Hernández Rivero, V.M., Santana Bonilla, P.J. y Sosa Alonso, J.J. (2021). Feedback y autorregulación del aprendizaje ēn educación superior. Revista de Investigación Educativa, 39(1), 227-248.

DOI: http://dx.doi.org/10.6018/rie.423341

\title{
Feedback y autorregulación del aprendizaje en educación superior
}

\author{
Feedback and self-regulated learning in higher education \\ Víctor M. Hernández Rivero, Pablo Joel Santana Bonilla y Juan José Sosa Alonso \\ Dpto. Didáctica e Investigación Educativa \\ Universidad de La Laguna \\ Tenerife-España
}

\begin{abstract}
Resumen
La evaluación formativa y el feedback se relacionan con la mejora del aprendizaje y su autorregulación. Estos procesos deben entenderse desde la interacción entre características personales, comportamentales y contextuales. Para que el feedback sea eficaz debe ser adecuadamente interpretado, reconstruido e internalizado. Este trabajo pretende analizar las percepciones y valoraciones del alumnado acerca del feedback recibido y sus efectos y relaciones sobre los procesos de autorregulación del aprendizaje (ARA). Para ello se aplicó un cuestionario a 603 estudiantes de siete asignaturas de seis titulaciones universitarias sobre sus percepciones y valoraciones del feedback recibido y se contrastaron con las características didácticas de las asignaturas en las que se desarrolla la recogida de información. A partir del análisis descriptivo y exploratorio de las respuestas al cuestionario se hipotetizaron relaciones entre las variables emergentes, que fueron sometidas a verificación mediante un modelo estructural, basado en mínimos cuadrados parciales (PLS-SEM). Los hallazgos confirman el modelo de relaciones hipotetizado a partir del estudio previo y sugieren que el alumnado construye una percepción positiva de feedback que ejerce una mayor influencia en sus procesos de ARA cuando se garantizan unos valores mínimos de los elementos siguientes: un feedback docente concurrente, oral, específico y centrado en la tarea, claro y comprensible; oportunidades para que se genere un feedback entre
\end{abstract}

Correspondencia: Víctor M. Hernández Rivero, vhernan@ull.edu.es, Dpto. Didáctica e Investigación Educativa, Facultad de Educación, Campus Central, Univ. La Laguna, Avda. Trinidad, s/n, 38204, La Laguna, Tenerife, España 
iguales dialógico e interactivo; el uso de las posibilidades que ofrecen los entornos virtuales para apoyar los procesos de feedback; y una clara especificación inicial de la tarea o del resultado de aprendizaje que se espera del alumnado.

Palabras clave: evaluación; retroalimentación; aprendizaje; educación.

\section{Abstract}

Formative assessment and feedback are related to learning improvement and self-regulation processes. These processes can be understood as the interplay between personal, behavioural and contextual characteristics. For feedback to be effective it must be properly interpreted, reconstructed and internalized. This article presents the results of a research project on feedback that aimed to achieve two purposes. The first was to describe and compare students' perception about the provided feedback and its relationship with the processes of self-regulated learning (SRA) in the different academic subjects of the project. The second sought to generate an exploratory model, based on PLS-SEM, about the relationships between the features of the received feedback, students' opinion about it, and its effects on SRA and students' satisfaction. A questionnaire was designed to measure students' perception of the feedback and was filled in by 603 students in seven subjects of six university degrees. The findings confirm the exploratory model generated and suggest that students construct a positive perception of feedback that exerts a greater influence on SRA when the following elements are present at a basic level: a concurrent teacher feedback, oral, specific and task-focused, clear and understandable; opportunities for the emergence of a dialogic and interactive peer-feedback; the use of the opportunities offered by virtual environments to give feedback; and a clear initial specification of the task or learning results expected (feed-up).

Keywords: assessment; feed-back; learning; education.

\section{Introducción}

La evaluación es uno de los elementos que más condiciona qué y cómo aprende el alumnado. Los estudiantes orientan sus actividades según las demandas del sistema de evaluación de la asignatura (Gibbs y Simpson, 2009). Por ello, la evaluación formativa y los procesos de feedback constituyen un ámbito de estudio preferente en la educación superior (Ajjawi \& Boud, 2015; Boud \& Molloy, 2015; Brown, 2015; Cano, 2014; Carless, 2016; Hattie \& Timperley, 2007; Margalef, 2014; Molloy \& Boud, 2015; Nicol, 2015).

La evaluación formativa se vincula con la mejora del aprendizaje porque ofrece al alumnado orientación en las actividades y tareas que realiza, facilita información sobre los procesos subyacentes y orientaciones sobre actitudes, relaciones y conducta; y por último, proporciona pautas sobre las estrategias que han sido empleadas para la autorregulación de su aprendizaje.

La autorregulación es un tópico de investigación complejo y ampliamente investigado (Boekaerts, Pintrich \& Zeidner, 2000; García-Jiménez, 2015; Salmerón, Gutierrez-Braojos, Rodriguez, y Salmeron-Vilchez, 2011). Es estudiada y entendida desde la interacción de procesos derivados de características personales, comportamentales y contextuales (Zimmerman, 2000). Depende de pensamientos auto-generados, sentimientos y acciones que se planifican y desarrollan, tratando de lograr los objetivos o fines personales, en un proceso de adaptación cíclica regulado por el contexto y la evolución personal. 
Nicol y MacFarlane-Dick (2006), basándose en Butler y Winne (1995), desarrollan esta relación entre los procesos de retroalimentación y el desarrollo de la autorregulación en el aprendizaje (ARA) en la educación superior (ver Figura 1).

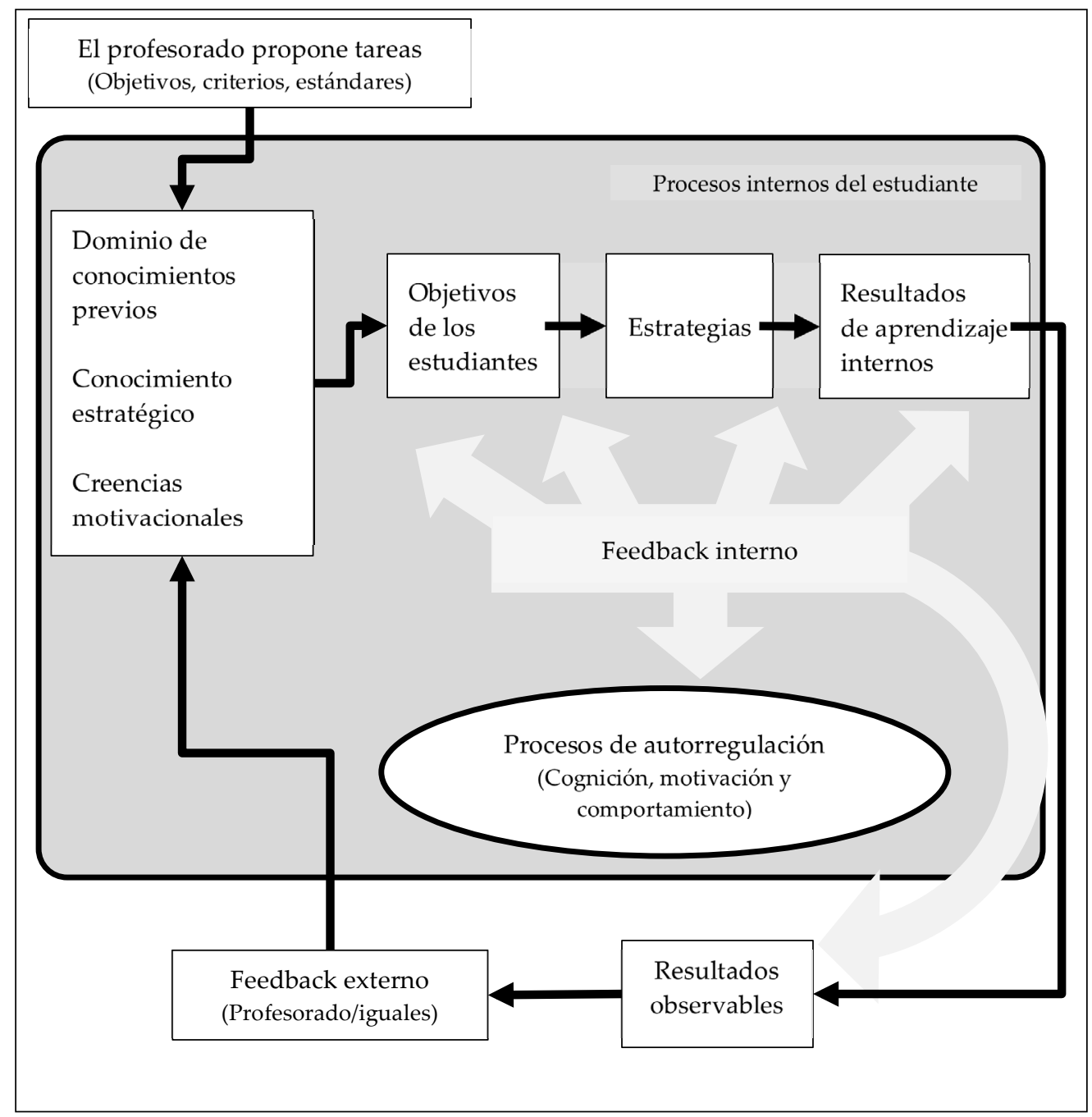

Figura 1. Adaptación del modelo de aprendizaje autorregulado propuesto por Nicol y MacFarlane-Dick (2006), a partir de Butler y Winne (1995).

El proceso parte de la tarea de aprendizaje que propone el profesorado. Este desencadenante pone en marcha los procesos de autorregulación internos del estudiante. El compromiso con la tarea requiere que el/la estudiante se base en conocimientos previos y creencias motivacionales y que construya una interpretación personal del significado de la tarea y de sus requerimientos. A partir de este conjunto de concepciones inter- 
nas, el alumnado formula sus propios objetivos de tarea. Aunque debería haber una superposición entre los objetivos del profesorado y el que genera el/la estudiante, el grado de superposición no tiene por qué ser muy alto. Los objetivos generados por el estudiantado podrían ser difusos, poco definidos o claros. En cualquier caso, estos objetivos acabarán definiendo las estrategias de aprendizaje por las que opte cada estudiante para alcanzar sus resultados, tanto internos como externos.

Los resultados internos hacen referencia a cambios cognitivos o afectivos que puedan producirse por haberse involucrado en la tarea. Los resultados observables son los productos tangibles que elaboran los estudiantes y las conductas que desarrollan como resultado de su trabajo en la tarea.

Un elemento clave del modelo son los procesos internos de control y gestión que cada estudiante moviliza, y que pone en relación los resultados que se van obteniendo con los objetivos planteados, y las estrategias y tácticas desplegadas. Estos mecanismos generan vías de feedback interno (Nicol \& MacFarlane-Dick, 2006) articulado sobre los tres niveles que definen los procesos de autorregulación: cognitivo, motivacional y comportamental. Es este feedback interno el que indica al estudiante si se está enfrentando a la tarea de forma adecuada o si debe introducir algún cambio.

A todo ello se añade el feedback externo que recibe el estudiante, facilitado por docentes, compañeros/as u otros medios (p.ej.: un/a tutor/a de prácticas o la mediación de un ordenador). Esta información adicional puede mejorar, reforzar o entrar en conflicto con la interpretación de la tarea por parte del alumnado y las previsiones de aprendizaje.

Este feedback externo se vincula al feedback interno para provocar efectos en los procesos internos y en los resultados de aprendizaje y, para ello, el feedback docente debe ser adecuadamente interpretado, reconstruido e internalizado.

Desde la perspectiva de Nicol y MacFarlane-Dick (2006), una buena práctica del feedback tiene que reforzar las capacidades del alumnado para autorregular su desempeño, atendiendo a las siguientes cualidades:

1) Clarifica qué es un "buen" desempeño en la tarea (objetivos de aprendizaje, criterios de evaluación, estándares esperados).

2) Facilita la auto-evaluación (reflexión) durante el aprendizaje.

3) Ofrece información de alta calidad sobre su aprendizaje.

4) Fomenta el diálogo con el profesorado y con los/las compañeros/as del grupo en torno al aprendizaje.

5) Promueve creencias motivadoras positivas y mejora la autoestima.

6) Ofrece oportunidades para equiparar rendimiento actual y deseado.

7) Proporciona información al profesorado para mejorar su enseñanza.

Este artículo aporta conocimiento acerca de las relaciones que se establecen entre los elementos y características del feedback externo (caracterizado por la identificación de los elementos objetivos, observables, que definen la vivencia de feedback que se ha producido en cada asignatura), el feedback interno (caracterizado por los pensamientos, valoraciones e interpretaciones que el alumnado va haciendo de la experiencia de enseñanza, evaluación y feedback que va recibiendo en cada asignatura), y los procesos de ARA que desarrolla el alumnado. 


\section{Método}

\section{Objetivos}

La investigación persigue los siguientes objetivos:

1) Describir y analizar las percepciones que tiene el alumnado acerca del feedback recibido (feedback externo) y contrastarlas con la descripción que hace el profesorado.

2) Establecer relaciones entre las características del feedback externo, los pensamientos, valoraciones e interpretaciones que genera (feedback interno) y los procesos de autorregulación del aprendizaje.

\section{Población y Muestra}

Han participado 603 estudiantes pertenecientes a siete asignaturas obligatorias de seis titulaciones de la Universidad de La Laguna (ver Tabla 1). Las edades abarcaban desde los 18 hasta los 57 años y la media era de 21,02 años. Un 28,4\% eran hombres y un $71,6 \%$ mujeres.

Tabla 1

Asignaturas y alumnado participante

\section{ASIGNATURAS, CURSO Y TITULACIÓN Siglas Créditos Estudiantes}

\begin{tabular}{|c|c|c|c|}
\hline $\begin{array}{l}\text { Organización de las Instituciones Educativas - 3ํㅡ Grado en } \\
\text { Pedagogía }\end{array}$ & OIE & 6 & 96 \\
\hline $\begin{array}{l}\text { Asesoramiento Institucional y Sistemas de Apoyo - } 3^{\underline{o}} \text { Grado } \\
\text { en Pedagogía }\end{array}$ & AIS & 6 & 143 \\
\hline $\begin{array}{l}\text { Percepción y Expresión Musical y su Didáctica - 3º Grado en } \\
\text { Maestro en Educación Infantil }\end{array}$ & PEM & 6 & 65 \\
\hline 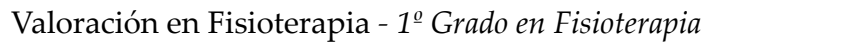 & VFI & 6 & 65 \\
\hline $\begin{array}{l}\text { Aprendizaje y Enseñanza de la Educación Física - Máster de } \\
\text { Formación del Profesorado de Secundaria }\end{array}$ & EAEF & 12 & 20 \\
\hline Planes de Formación - 2º Grado en Pedagogía & $\mathrm{PFO}$ & 6 & 70 \\
\hline $\begin{array}{l}\text { Sociedad, Familia y Escuela, - 1ํㅡ Grado en Maestro Educación } \\
\text { Primaria }\end{array}$ & SFE & 6 & 144 \\
\hline
\end{tabular}

\section{Instrumento}

El cuestionario sobre feedback se construyó a partir de una amplia revisión bibliográfica y el análisis de los siguientes instrumentos: 
- Assessment Experience Questionnaire-AEQ (Gibbs \& Simpson, 2003; Núñez \& Reyes, 2014);

- Motivated Strategies for Learning Questionnaire (Pintrich \& DeGroot, 1990);

- Cuestionario ActEval (Quesada, Rodríguez-Gómez \& Ibarra, 2013);

- Cuestionario para la Evaluación de las estrategias de Aprendizaje de los Estudiantes Universitarios-CEVEAPEU (Gargallo, Suárez-Rodríguez \& Pérez-Pérez, 2009).

La selección de ítems y construcción del cuestionario se planteó desde una perspectiva teórico-deductiva, tratando de que, en su conjunto, el instrumento permitiera recabar información sobre los elementos definitorios de la vivencia del feedback externo, los pensamientos, valoraciones e interpretaciones que definen el feedback interno y, finalmente, elementos relacionados con la ARA.

El cuestionario, quedó organizado en cinco dimensiones y seis subdimensiones: feed-up, feed-back, autoevaluación, autorregulación y satisfacción del alumnado (ver Apéndice 1). La escala de respuesta se organizaba en 5 niveles, oscilando entre el valor "nada" (1) y "mucho" (5).

El cuestionario se sometió a un proceso de revisión por parte de diez expertos, docentes universitarios, con amplia experiencia en el ámbito de la evaluación formativa. El acuerdo interjueces aconsejó mantener la mayoría de las cuestiones en su primera redacción y añadir matices en el caso de ítems en los que la redacción se juzgó dudosa o con interpretación equívoca.

\section{Procedimiento de recogida y análisis de datos}

Se recopiló información acerca del feedback percibido por el alumnado mediante un cuestionario on line en el aula virtual de cada asignatura, aplicado entre 2018 y 2019, una vez desarrollada la tarea elegida para esta investigación.). También se recogió información sobre el feedback planificado por cada docente, con el objeto de contextualizar el feedback percibido por el alumnado y extraer conclusiones más ajustadas (ver Tabla 2).

Tabla 2

Contextualización didáctica de las tareas

\begin{tabular}{|c|c|c|c|c|c|c|c|c|}
\hline \multirow{2}{*}{$\begin{array}{l}\text { DIMENSIONES } \\
\text { DEL FEED-BACK }\end{array}$} & \multirow{2}{*}{$\begin{array}{l}\text { SUBDIMENSIONES } \\
\text { DEL FEED-BACK }\end{array}$} & \multicolumn{7}{|c|}{ TAREAS SEGÚN ASIGNATURAS } \\
\hline & & OIE & AIS & PEM & VFI & EAEF & PFO & SFE \\
\hline \multirow{3}{*}{$\begin{array}{l}\text { 1. Agentes que lo } \\
\text { proporcionan }\end{array}$} & El profesor/la profesora & * & $*$ & * & * & * & * & * \\
\hline & Compañeros/as & & & & & & * & * \\
\hline & Compañeros/as de grupo & * & * & * & * & * & * & * \\
\hline \multirow{3}{*}{ 2. Destinatarios } & Individual & & & * & & & & \\
\hline & Pequeño grupo & * & $*$ & & * & * & * & \\
\hline & Grupo-clase & * & * & * & * & & * & * \\
\hline
\end{tabular}




\begin{tabular}{|c|c|c|c|c|c|c|c|c|}
\hline \multirow{2}{*}{$\begin{array}{l}\text { DIMENSIONES } \\
\text { DEL FEED-BACK }\end{array}$} & \multirow{2}{*}{$\begin{array}{l}\text { SUBDIMENSIONES } \\
\text { DEL FEED-BACK }\end{array}$} & \multicolumn{7}{|c|}{ TAREAS SEGÚN ASIGNATURAS } \\
\hline & & OIE & AIS & PEM & VFI & EAEF & PFO & SFE \\
\hline \multirow{4}{*}{$\begin{array}{l}\text { 3. Medios de } \\
\text { provisión }\end{array}$} & Oral & * & * & & * & * & * & $*$ \\
\hline & Escrito en papel & & & * & & & & \\
\hline & Escrito digitalizado & & & & & & * & * \\
\hline & $\begin{array}{l}\text { Gráfico, por medio de } \\
\text { rúbrica }\end{array}$ & & * & * & * & & * & $*$ \\
\hline \multirow{3}{*}{$\begin{array}{l}\text { 4. Momento de } \\
\text { provisión }\end{array}$} & Concurrente & * & * & & * & & * & \\
\hline & Justo al terminar la tarea & & & * & & & * & $*$ \\
\hline & Retardada & & & & & & & \\
\hline
\end{tabular}

5. Previsión de mejora de la tarea entregada por alumnado

\begin{tabular}{|c|c|c|c|c|c|c|c|c|}
\hline \multirow{4}{*}{$\begin{array}{l}\text { 6. Tipos de } \\
\text { feedback }\end{array}$} & Centrado en la tarea & * & * & * & * & * & * & * \\
\hline & Centrado en el proceso & & * & * & & * & & \\
\hline & $\begin{array}{l}\text { Centrado en la } \\
\text { autorregulación }\end{array}$ & & * & & & & & * \\
\hline & $\begin{array}{l}\text { Centrado en la valoración } \\
\text { personal }\end{array}$ & & & & & & & \\
\hline \multirow{2}{*}{$\begin{array}{l}\text { 7. Orientación } \\
\text { pasada/futura de } \\
\text { la información que } \\
\text { incluye }\end{array}$} & Retrospectivo (feedback) & * & * & * & & & * & \\
\hline & $\begin{array}{l}\text { Acción futura } \\
\text { (feed-forward) }\end{array}$ & * & & & * & & * & \\
\hline \multirow{4}{*}{$\begin{array}{l}\text { 8. Presencia de } \\
\text { feed-up }\end{array}$} & $\begin{array}{l}\text { Especificación consigna } \\
\text { inicial }\end{array}$ & * & * & * & * & & * & * \\
\hline & $\begin{array}{l}\text { Vinculación con } \\
\text { competencias }\end{array}$ & & * & * & & & * & \\
\hline & $\begin{array}{l}\text { Vinculación con criterios } \\
\text { de calificación de la } \\
\text { asignatura }\end{array}$ & & * & & & & * & * \\
\hline & $\begin{array}{l}\text { Vinculación con conteni- } \\
\text { dos de la asignatura }\end{array}$ & * & & & * & & * & \\
\hline \multicolumn{2}{|c|}{ 9. Previsión de feedback dialógico } & & * & & * & & & * \\
\hline \multicolumn{2}{|c|}{ 10. Feedback acompañado de calificación } & * & & & & & * & * \\
\hline \multicolumn{2}{|c|}{$\begin{array}{r}\text { Elementos conformantes o definitorios de } \\
\text { feedback }\end{array}$} & 12 & 15 & 12 & 12 & 6 & 18 & 14 \\
\hline
\end{tabular}


Para recopilar la información detallada en la tabla 2 se solicitó a cada docente responsable de las asignaturas participantes que cumplimentara una ficha didáctica, referida al feedback en la tarea sobre la que se preguntaría al alumnado en el cuestionario.

Para el análisis cuantitativo, dado que el volumen de datos perdidos no excedía en ningún caso el 5\%, se trataron mediante el método de sustitución basada en la media. En general, se ha optado por realizar análisis con estadísticos no paramétricos o, en su defecto, estadísticos paramétricos considerados robustos al incumplimiento de supuestos.

El análisis de datos se ha desarrollado en 2 fases. En la primera fase, relacionada con el primer objetivo de investigación, se ha procedido al análisis de asociación (rho de Spearman) entre los ítems integrados en las diferentes dimensiones del cuestionario (ver Apéndice 1) y al análisis de componentes principales sobre los 22 ítems orientados a determinar la percepción de los elementos descriptivos del feedback externo buscando hacer emerger aspectos definitorios de la "percepción" del feedback del alumnado que faciliten su interpretación. El resto de ítems no se incluyen en este análisis por constituir aspectos definitorios de lo que Nicol y McFarlane-Dick (2006) identifican como elementos del "feedback interno", que interviene en análisis posteriores.

A partir del agrupamiento de las variables resultantes del análisis de componentes principales, se ha realizado un análisis comparativo (Kruskal-Wallis) entre las diversas asignaturas participantes. El tamaño del efecto se ha estimado por medio del índice (2) (Snyder \& Lawson, 1993).

Los resultados obtenidos se han interpretado recurriendo a las diferencias encontradas entre las características y peculiaridades didácticas de cada asignatura (ver Tabla 2).

Para el logro del segundo objetivo se partió de las relaciones hipotetizadas entre las características descriptivas del feedback externo, ofrecido en las diferentes asignaturas y las variables "valoración del feedback", "autorregulación del aprendizaje", "satisfacción con el sistema de evaluación" y "satisfacción con el aprendizaje", surgidas del análisis de componentes principales. Las hipótesis se sometieron a prueba mediante un modelo de ecuaciones estructurales basado en el método de mínimos cuadrados parciales (PLS-SEM) (Hair, Hult, Ringle \& Sarstedt, 2017) que se describe en la Figura 3.

La evaluación y análisis del modelo PLS-SEM obtenido se ha basado, siguiendo las recomendaciones establecidas por Hair et al. (2017), en el análisis del modelo de medida, por un lado, y del modelo estructural, por otro.

Todos los análisis se han realizado con ayuda del paquete estadístico SPSS, versión 25.0.0. y del programa SmartPLS, versión 3.

\section{Resultados}

En relación al primer objetivo de investigación, tratando de agrupar variables descriptivas de las características del feedback externo percibido por el alumnado en sus asignaturas, y poder así establecer comparaciones con las descripciones que hacía el profesorado, se decidió aplicar un análisis de componentes principales (KMO=0,900; Test de Esfericidad de Bartlett $\mathrm{p}<, 000)$ sobre los ítems relacionados con la percepción del tipo y características de feedback desarrollado en clase (22 ítems relacionados 
con la descripción del feedback externo). En Tabla 3 se reproduce el resultado de la convergencia del análisis del que emergen 4 componentes que explican el $53 \%$ de la varianza observada.

Tabla 3

Análisis de componentes principales de los 22 items sobre características del feedback percibido ${ }^{1}$

\begin{tabular}{|c|c|c|c|c|c|c|}
\hline \multicolumn{7}{|c|}{ Matriz de componente rotados } \\
\hline \multirow{2}{*}{ ITEMS } & \multicolumn{4}{|c|}{$\begin{array}{l}\text { Pesos factoriales en cada } \\
\text { Componente }\end{array}$} & \multirow{2}{*}{$\begin{array}{l}\text { Interpretación del } \\
\text { componente }\end{array}$} & \multirow{2}{*}{$\begin{array}{l}\text { Alfa de } \\
\text { Cronbach }\end{array}$} \\
\hline & 1 & 2 & 3 & 4 & & \\
\hline
\end{tabular}

17. He recibido feedback del profesor o profesora

0,755

8. Recibí feedback de manera oral de modo presencial

0,735

3. Recibimos feedback como grupo pequeño

0,733

6. Recibimos feedback todo el grupo clase

0,708

25. Recibí feedback en el plazo acordado

0,693

40. He recibido feedback sobre contenidos de la tarea que debía ampliar o mejorar

1. He recibido feedback sobre estrategias de trabajo o habilidades que necesitaba desarrollar

Feedback centrado en la figura del profesor, provisto de manera oral presencial a todo el grupo clase o en grupos, centrado en tareas y destrezas para desarrollarlas y aplicado de manera concurrente. FEEDBACK DOCENTE

23. He recibido feedback durante la realización de la tarea

0,638

42. He recibido feedback después de entregar el 0,588 primer borrador

22. Recibí feedback por medio de preguntas que me hicieron reflexionar 


\begin{tabular}{lllllll}
\hline \multicolumn{3}{c}{ Matriz de componente rotados } \\
\hline ITEMS & \multicolumn{2}{c}{$\begin{array}{c}\text { Pesos factoriales en cada } \\
\text { Componente }\end{array}$} & $\begin{array}{c}\text { Interpretación del } \\
\text { componente }\end{array}$ & $\begin{array}{c}\text { Alfa de } \\
\text { Cronbach }\end{array}$ \\
\cline { 2 - 5 } & 1 & 2 & 3 & 4 & & \\
\hline
\end{tabular}

30. He recibido feedback de un compañero o 0,699 compañera

16. He recibido feedback de grupos de compañeros y $\quad 0,615$ compañeras

35. He recibido feedback sobre aspectos personales (mensajes de ánimo, 0,601 valoraciones sobre capacidades...)

4. He tenido la oportunidad de ofrecer feedback a mis compañeros y compañeras de clase 0,594 y ellos/as conocían mi identidad

46. Recibí feedback individualmente 0,583

38. Recibí feedback por escrito en papel en mano

13. He tenido la oportunidad de ofrecer feedback de forma anónima a mis compañeros y compañeras de clase

31. Recibí feedback de modo gráfico (sombreando en una rúbrica...)

32. El feedback fue acompañado de la calificación

45. He recibido feedback una vez entregada 0,558 definitivamente la tarea
0,728

0,748

Feedback por medio de rúbricas, sumativo $y$ final

FEEDBACK RÚBRICAS
DBACK ENTRE

\section{IGUALES}




\begin{tabular}{|c|c|c|c|c|c|c|}
\hline \multicolumn{7}{|c|}{ Matriz de componente rotados } \\
\hline \multirow{2}{*}{ ITEMS } & \multicolumn{4}{|c|}{$\begin{array}{c}\text { Pesos factoriales en cada } \\
\text { Componente }\end{array}$} & \multirow{2}{*}{$\begin{array}{l}\text { Interpretación del } \\
\text { componente }\end{array}$} & \multirow{2}{*}{$\begin{array}{l}\text { Alfa de } \\
\text { Cronbach }\end{array}$} \\
\hline & 1 & 2 & 3 & 4 & & \\
\hline $\begin{array}{l}\text { 2. Recibí feedback por } \\
\text { escrito a través de e-mail } \\
\text { o del aula virtual }\end{array}$ & & & & 0,684 & \multirow{2}{*}{$\begin{array}{c}\text { Feedback telemático, } \\
\text { impersonal } \\
\text { FEEDBACK } \\
\text { TELEMÁTICO }\end{array}$} & \multirow{2}{*}{0,603} \\
\hline $\begin{array}{l}\text { 43. Recibí feedback de } \\
\text { modo oral on line (aula } \\
\text { virtual, WhatsApp...) }\end{array}$ & & & & 0,678 & & \\
\hline \multicolumn{3}{|c|}{${ }^{1}$ Extracción eigen $>=1$, rotación Varimax } & \multicolumn{3}{|c|}{ Total de la escala } & 0,885 \\
\hline
\end{tabular}

Se identificaron cuatro características del feedback externo: feedback docente, feedback entre iguales, feedback mediante rúbricas y feedback telemático. Dichos factores parecen resumir la percepción de las características "objetivas" del feedback en las diferentes asignaturas (feedback externo). La Figura 2 ilustra la presencia de los componentes de feedback obtenidos del análisis anterior en cada asignatura, superponiendo la valoración del feedback externo y de sus efectos sobre la ARA.

La interpretación de la Figura 2 permite suponer que existen relaciones interactivas complejas entre las características del feedback, la valoración que el alumnado hace del feedback y sus efectos sobre la ARA (hipótesis 1).

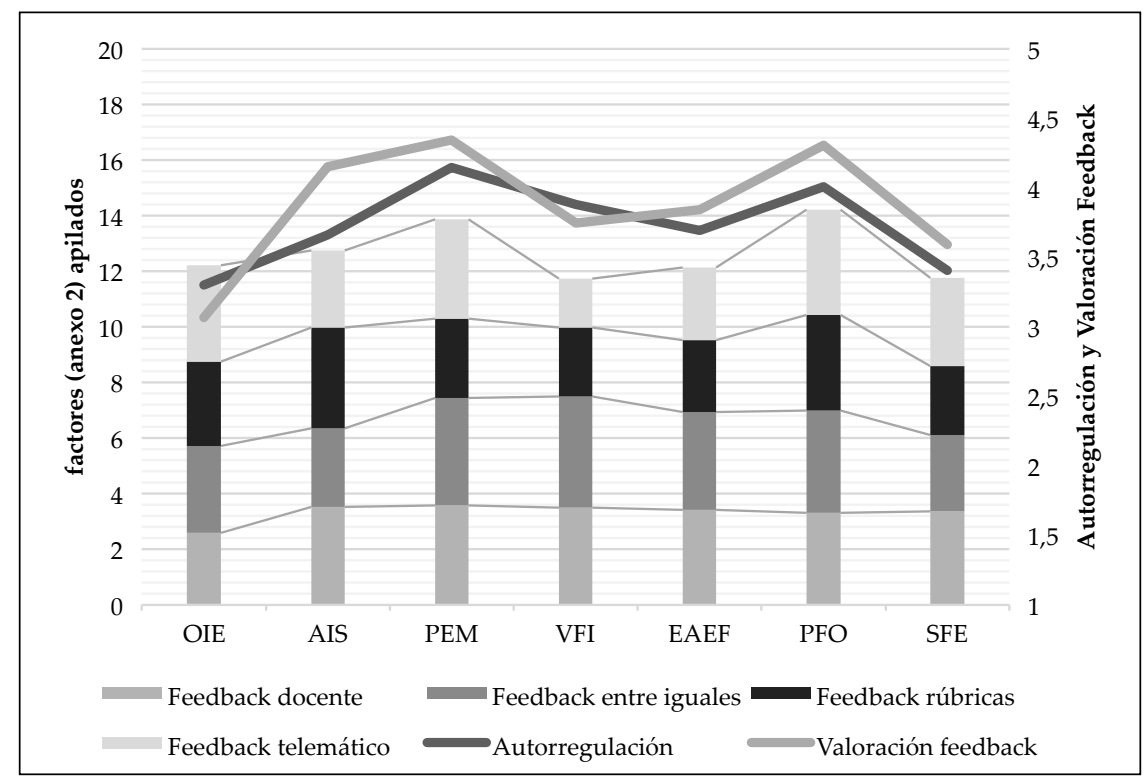

Figura 2. Presencia, por asignaturas, de las características del feedback, su valoración y efectos sobre la autorregulación en el aprendizaje según la percepción del alumnado. 
Tabla 4.

Resultados del análisis de varianza no paramétrico ( $H$ de Kruskal-Wallis) para cada uno de los componentes emergidos del análisis componentes principales y la valoración del feed-back, entre asignaturas.

\begin{tabular}{cccccccccccc}
\hline & \multicolumn{1}{c}{} & \multicolumn{4}{c}{ Asignaturas } & \multicolumn{4}{c}{ Kruskal-Wallis } \\
\cline { 2 - 11 } Constructos & OIE & AIS & PEM & VFI & EAEF & PFO & SFE & H & P valor & $\varepsilon^{2}$ \\
\hline Feedback docente & 2,59 & 3,53 & 3,58 & 3,50 & 3,43 & 3,32 & 3,36 & 18,65 & 0,005 & 0,06 \\
\hline Feedback entre iguales & 3,13 & 2,82 & 3,86 & 4,00 & 3,51 & 3,68 & 2,75 & 70,64 & 0,000 & 0,22 \\
\hline Feedback rúbricas & 3,03 & 3,62 & 2,86 & 2,47 & 2,59 & 3,44 & 2,47 & 67,22 & 0,000 & 0,21 \\
\hline Feedback telemático & 3,47 & 2,79 & 3,58 & 1,76 & 2,61 & 3,77 & 3,19 & 66,55 & 0,000 & 0,20 \\
\hline Valoración feedback & 3,07 & 4,15 & 4,34 & 3,75 & 3,84 & 4,31 & 3,59 & 43,50 & 0,000 & 0,13 \\
\hline Autorregulación & 3,30 & 3,66 & 4,15 & 3,88 & 3,69 & 4,01 & 3,41 & 41,45 & 0,000 & 0,15 \\
\hline
\end{tabular}

Aunque todas las diferencias entre asignaturas son estadísticamente significativas, los tamaños de los efectos son pequeños. El factor feedback docente no genera diferencias entre las asignaturas $\left(\varepsilon^{2}=0,06\right)$. Sin embargo, se observa que cuando no está presente con una intensidad equiparable a las del resto de asignaturas (caso de OIE), afecta a la valoración que hace el alumnado de la percepción de feedback generado en clase (hipótesis 2). Ninguna de las variantes de feedback identificadas parece ser, por sí sola, suficiente para explicar la valoración que hace el alumnado. Más bien, parece que es la conjunción de diferentes características del feedback la que acaba generando una impresión más positiva en el alumnado y una percepción de mayor efecto sobre la ARA (hipótesis 3).

En relación al segundo objetivo de investigación, que pretende explorar la plausibilidad de las relaciones interactivas que se hipotetizan, a partir del análisis previo, entre los factores obtenidos y las variables objetivo u endógenas, se generó un modelo PLSSEM. La expresión gráfica del modelo, una vez obtenidas las cargas, los coeficientes path y las $\mathrm{R}^{2}$ de las variables latentes incorporadas en el modelo, se muestra en la Figura 3.

El resultado del análisis tetrad confirmatorio (Gudergan, Ringle, Wende \& Will, 2008) valida las decisiones adoptadas, en relación al modelo de medida (formativa/ reflectiva) elegido para cada variable endógena y exógena.

En general, para todos los constructos incluidos en el modelo, generados sobre una base de medida reflectiva, satisfacen los criterios de consistencia interna, de validez convergente y de validez discriminante.

En el caso del constructo ARA, con modelo de medida formativo, se han descartado problemas de colinealidad y se ha verificado que los pesos con los que se vinculan los indicadores asociados con el constructo ARA son relevantes y significativamente distintos de 0 .

La estimación de los valores path (error típico de medida estimado mediante bootstrapping con 5000 sub-muestras derivadas y error tolerado $<5 \%$ ) aparece representada en la Figura 3. 


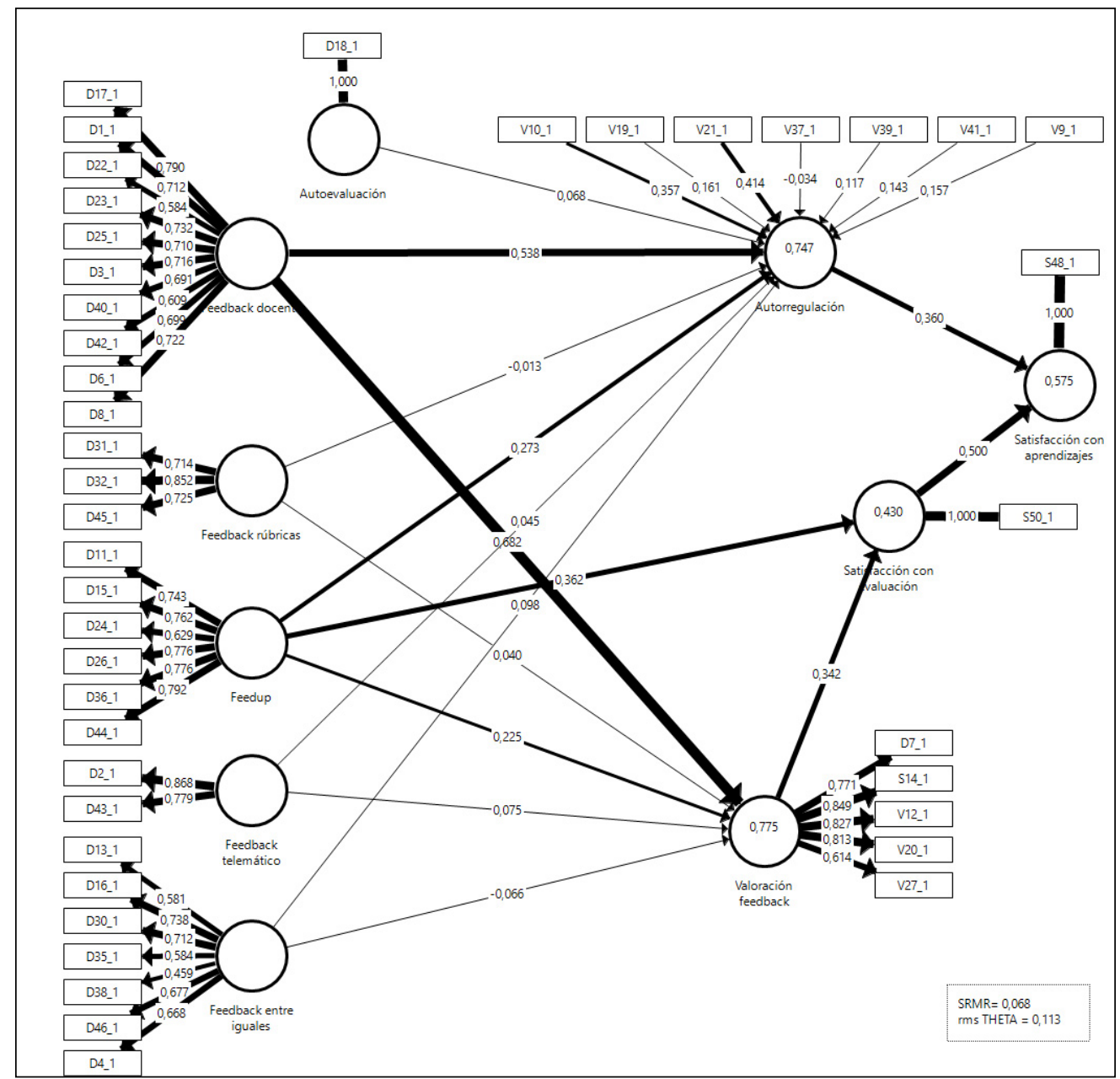

Figura 3. Representación del modelo PLS-SEM generado (en trazo más grueso se destacan las relaciones significativas distintas de 0 ).

Finamente, el análisis de los efectos totales (suma de efectos directos e indirectos) de cada uno de los constructos en las diferentes relaciones hipotetizadas permitió adoptar la decisión de mantener en el modelo todas las variables exógenas y endógenas inicialmente contempladas, con la estructura de relaciones intacta, tratando de preservar los efectos indirectos sobre las variables latentes.

La Tabla 5 presenta un resumen del análisis realizado a partir del modelo estructural generado, en términos de varianza explicada $\left(R^{2}\right)$ y capacidad predictiva del modelo $\left(Q^{2}\right)$ para cada una de las variables endógenas contempladas. También se muestran los valores de los coeficientes path, los tamaños del efecto $\left(\mathrm{f}^{2}\right)$ y las capacidades predictivas $\left(\mathrm{q}^{2}\right)$ de las variables endógenas y exógenas. 
Tabla 5

Valores obtenidos en el proceso de validación del modelo estructural generado

\begin{tabular}{|c|c|c|c|c|c|c|c|c|c|c|c|c|c|c|}
\hline \multirow{2}{*}{\multicolumn{3}{|c|}{$\begin{array}{l}\text { Variables endógenas } \\
\qquad \mathrm{SRMR}=0.068 \\
\mathrm{RMR}_{\text {theta }}=0,113\end{array}$}} & \multicolumn{3}{|c|}{ Autorregulación } & \multicolumn{3}{|c|}{$\begin{array}{l}\text { Valoración del } \\
\text { feedback }\end{array}$} & \multicolumn{3}{|c|}{$\begin{array}{c}\text { Satisfacción con la } \\
\text { evaluación }\end{array}$} & \multicolumn{3}{|c|}{$\begin{array}{l}\text { Satisfacción con el } \\
\text { aprendizaje }\end{array}$} \\
\hline & & & path & $\mathrm{f}^{2}$ & $\mathrm{q}^{2}$ & path & $\mathrm{f}^{2}$ & $\mathrm{q}^{2}$ & path & $\mathrm{f}^{2}$ & $\mathrm{q}^{2}$ & path & $\mathrm{f}^{2}$ & $\mathrm{q}^{2}$ \\
\hline \multirow{4}{*}{ 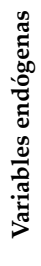 } & \multicolumn{2}{|c|}{ Autorregulación } & & & & & & & & & & 0,36 & 0,22 & 0,17 \\
\hline & \multicolumn{2}{|c|}{$\begin{array}{l}\text { Valoración del } \\
\text { feedback }\end{array}$} & & & & & & & 0,34 & 0,10 & 0,09 & & & \\
\hline & \multicolumn{2}{|c|}{$\begin{array}{l}\text { Satisfacción con } \\
\text { la evaluación }\end{array}$} & & & & & & & & & & 0,50 & 0,41 & 0,33 \\
\hline & \multicolumn{2}{|c|}{$\begin{array}{l}\text { Satisfacción con } \\
\text { el aprendizaje }\end{array}$} & & & & & & & & & & & & \\
\hline \multirow{8}{*}{ 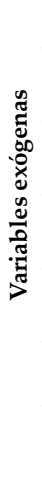 } & \multirow{2}{*}{\multicolumn{2}{|c|}{$\begin{array}{l}\text { Autoevaluación } \\
\begin{array}{l}\text { Feedback } \\
\text { docente }\end{array} \\
\end{array}$}} & 0 & 0 & 0 & & & & & & & & & \\
\hline & & & 0,54 & 0,49 & 0,07 & 0,68 & 0,90 & 0,21 & & & & & & \\
\hline & \multicolumn{2}{|c|}{$\begin{array}{l}\text { Feedback entre } \\
\text { iguales }\end{array}$} & 0,10 & 0,02 & 0,02 & 0 & 0 & 0 & & & & & & \\
\hline & \multicolumn{2}{|l|}{$\begin{array}{l}\text { Feedback } \\
\text { rúbricas }\end{array}$} & 0 & 0 & 0 & 0 & 0 & 0 & & & & & & \\
\hline & \multicolumn{2}{|l|}{$\begin{array}{l}\text { Feedback } \\
\text { telemático }\end{array}$} & 0 & 0 & 0 & 0,08 & 0 & 0 & & & & & & \\
\hline & \multicolumn{2}{|l|}{ Feedup } & 0,27 & 0,11 & 0,02 & 0,23 & 0,09 & 0,02 & 0,36 & 0,11 & 0,10 & & & \\
\hline & & $\mathbf{R}^{2}$ & & 0,75 & & & 0,78 & & & 0,43 & & & 0,58 & \\
\hline & & $\mathrm{Q}^{2}$ & & 0,34 & & & 0,44 & & & 0,42 & & & 0,55 & \\
\hline
\end{tabular}

Los valores "0" reflejados en la Tabla 5 representan que el valor obtenido no es, estadísticamente, diferente de $0 \mathrm{o}$, en el caso de $\mathrm{f}^{2} \mathrm{y} \mathrm{q}^{2}$, que no alcanza el valor mínimo de 0,02 .

Los valores obtenidos indican que los niveles de varianza explicada $\left(R^{2}\right)$ por el modelo, en el caso de las variables latentes autorregulación y valoración del feedback es importante (por encima de 0,7). En el resto de los casos la varianza explicada es moderada.

A partir de los valores $\mathrm{Q}^{2}$ detallados en la Tabla 5, podemos afirmar que el modelo aporta relevancia predictiva en relación a todas las variables endógenas presentes en el modelo, al estar todos los valores $\mathrm{Q}^{2}$ claramente por encima de 0 .

Tomando como referentes los criterios generales de bondad de ajuste del modelo (Lohmöller, 1989), hemos obtenido un SRMR $=0,068$ y un $\mathrm{RMS}_{\text {theta }}=0,113$, ambos por debajo del umbral de tolerancia máxima recomendado de 0,08 y 0,12, respectivamente (Hair et al., 2017). 


\section{Discusión y conclusiones}

En relación al primer objetivo de investigación, la indagación en las percepciones del feedback y las relaciones de éste con los procesos de ARA, se han identificado cuatro tipos de feedback externo, según la descripción del alumnado en los entornos didácticos analizados.

El primer tipo tiene que ver con la provisión de feedback por parte del docente, acompañado de una serie de factores identificados como elementos de calidad en el feedback (inmediato, específico, orientado a la tarea y concurrente), y que hemos identificado como "feedback docente" con características similares a las identificadas por Hattie y Timperley (2007) como "feedback de calidad".

El segundo tipo se articula a partir de las relaciones interactivas entre el alumnado, "feed-back entre iguales", que se producen en el entorno de clase o fuera de ella, espontáneas o planificadas por los docentes, que ofrecen una retroalimentación complementaria, añadiendo elementos que trascienden la tarea para incluir aspectos personales. La importancia de este tipo de interacciones ya fue descrita por Boud y Molloy (2015), Cano (2014), Ladyshewsky (2015), y Sánchez-Martí, Muñoz y Ion (2019).

Un tercer tipo es la provisión de feedback por medio de rúbricas, adoptando en la mayoría de los casos un carácter formal y finalista. La rúbrica tiene la ventaja de que ofrece una información detallada por escrito en relación a los estándares de ejecución esperados, pero una de sus limitaciones, tal como se ha utilizado en las asignaturas del proyecto, es un instrumento cerrado y, con frecuencia, poco familiar y comprensible para el alumnado. Una alternativa la constituyen las rúbricas construidas colaborativamente.

Finalmente, se identificó un cuarto tipo relacionado con el feedback facilitado a través de medios telemáticos (aula virtual, correo electrónico o whatsapp). En el caso de las asignaturas estudiadas, vinculado a la co-evaluación y evaluación compartida.

Cuando se cotejan las percepciones del alumnado (ver Figura 2) con la descripción que realiza el profesorado del feedback que han pretendido ofrecer en el aula (Tabla 3) aparecen contrastes notables. El análisis de varianza no paramétrico (H de Kruskal- Wallis) realizado desvela que el alumnado apenas percibe diferencias importantes en el feedback que se ha desarrollado entre asignaturas, lo que no coincide con las descripciones realizadas por el profesorado, que se muestran bastante más heterogéneo. Por otra parte, el alumnado parece detectar cualidades de feedback que, en principio no estaba previsto desarrollar y otras que, estando previstas, pasan desapercibidas en algunos casos por el alumnado. Así, por ejemplo, en todas las asignaturas estaba prevista la existencia de un feedback docente, pero este no parece haber sido percibido de igual modo por el alumnado. O, en el caso de la asignatura SFE, según la descripción docente el feedback entre iguales se pretendía especialmente intenso (entre compañeros de gran grupo y de pequeño grupo) y sin embargo, la valoración que hace el alumnado indica una presencia escasa de esta modalidad de feedback.

Este resultado sugiere que, en futuras investigaciones, se debe profundizar en el análisis de las percepciones del alumnado acerca del feedback recibido, cotejándolas con el feedback docente efectivamente ofrecido. Esto es congruente con el modelo de feedback descrito por Nicol y MacFarlane-Dick (2006), que alude a los procesos de 
feedback interno versus externo y la necesidad de conocer las interpretaciones que hace el alumnado acerca del feedback externo recibido.

El análisis comparativo de las percepciones de feedback del alumnado apunta que los diferentes factores que configuran la percepción de feedback se relacionan entre sí de manera aditiva. La valoración es más positiva, tanto en lo que se refiere al feedback recibido como a los efectos percibidos sobre la ARA, en la medida en que hay una valoración más alta en cada uno de los cuatro factores (ver Figura 2). Las relaciones entre los factores parecen mantener, además, cierto carácter interactivo sobre la valoración del feedback y la percepción efectos sobre la ARA.

Los resultados obtenidos permiten destacar la importancia del factor "feedback docente" como factor clave en la percepción de la calidad del feedback y sobre la autorregulación del aprendizaje. Esta importancia no procede de su mera presencia, bastante similar en todas las asignaturas, sino más bien en el efecto negativo de su ausencia. Es decir, cuando el alumnado considera que este tipo de feedback no es suficiente la valoración general que se hace del feedback y de sus efectos sobre la ARA decaen. En esta línea de análisis, a partir de un valor mínimo en el factor "feedback docente", parecería que la otra variable que, en mayor medida parece afectar a la valoración del feedback es la existencia de un "feedback telemático". En cualquier caso, los diferentes tipos de percepción de feedback parecen mantener relaciones complejas entre sí, de carácter aditivo e interactivo, afectando tanto a la valoración que hace el alumnado del feedback como a sus procesos de ARA. Estas relaciones complejas son las que han dado lugar al establecimiento de las 3 hipótesis que se someten a prueba en el marco del objetivo 2 de este trabajo.

El modelo PLS-SEM generado ofrece un marco interpretativo que permite someter a prueba las hipótesis establecidas y explicar mejor algunos de los fenómenos interactivos descritos anteriormente.

Por un lado, se ha confirmado la trascendencia del "feedback docente", pero se añade, como elemento clave en la valoración positiva del feedback y su trascendencia sobre la ARA, la existencia de un buen "feed-up". Además, se observa que el "feedback por medio de rúbricas" no afecta de manera significativa ni a la valoración que se hace del feedback, ni a los efectos sobre la ARA. Por su parte, el "feedback entre iguales" ha demostrado tener una relación limitada con los procesos de autorregulación sobre el aprendizaje y prácticamente nula en relación con la valoración positiva del feedback. Al contrario de lo que sucede con el "feedback facilitado por medios telemáticos", que parece afectar levemente a la valoración positiva de la percepción de feedback pero no mantiene relación alguna con los procesos de ARA. El impacto del "feedback telemático" sobre los procesos de autorregulación sobre el aprendizaje debe ser tomado con cautela porque puede estar condicionado por factores como la escasa presencia de este tipo de feedback en las asignaturas analizadas, la falta de calidad del feedback ofrecido por dichos medios, o por carencias de formación del alumnado para utilizar o sacar provecho de este tipo de feedback.

Se ha constatado que la valoración del feedback y la percepción de ARA mantienen una relación positiva, aunque moderada, con la satisfacción del alumnado con la evaluación. A su vez, la satisfacción con la evaluación tiene una relación también moderada con la satisfacción con los aprendizajes. Este resultado resulta coherente 
por cuanto lo esperable es que la satisfacción relativa a constructos tan complejos y globales como la evaluación y el aprendizaje se vea afectada por otras variables que no han sido contempladas en el modelo utilizado.

En síntesis, las relaciones interactivas hipotetizadas a partir de los resultados obtenidos en la primera parte de este estudio (ver Figura 2), y exploradas en el modelo estructural generado (Figura 3), permiten indicar que el alumnado construye una percepción positiva de feedback que influye en mayor medida a sus procesos de ARA cuando los elementos siguientes están simultáneamente presentes:

1) Un feedback docente concurrente, oral, específico y centrado en la tarea, claro y comprensible.

2) Oportunidades para que se genere un feedback entre iguales dialógico e interactivo.

3) Uso de las posibilidades que ofrecen los entornos virtuales para apoyar los procesos de feedback.

4) Una clara especificación inicial de la tarea o resultado de aprendizaje que se espera del alumnado.

Estos resultados se alinean con los obtenidos por Nicol y MacFarlane-Dick (2006).

El análisis del feedback en la docencia universitaria es un tema de investigación con larga tradición en otros países de nuestro entorno, sin embargo, en España es de más reciente interés. La investigación sobre feedback partió de un constructo global que se ha ido concretando y perfilando, tanto conceptual como metodológicamente. Eso ha permitido abrir nuevas vías de análisis y profundización en relación a dicho fenómeno. Ejemplo de ello es el tema de este artículo, donde se profundiza en las relaciones entre feedback y autorregulación del aprendizaje por parte del alumnado.

Los resultados de este estudio evidencian la necesidad de seguir profundizando en un tema tan complejo, en el que intervienen muchas variables que interactúan entre sí. Si se quiere mejorar la docencia universitaria se debe apostar por modelos de enseñanza más centrados en el alumnado y en la calidad de sus aprendizajes, modelos en los que el feedback y su relación con la autorregulación del aprendizaje juegan un papel fundamental.

\section{Referencias}

Ajjawi, R. \& Boud, D. (2015). Researching feedback dialogue: an interactional analysis approach. Assessment E Evaluation in Higher Education 42(2), 252-265. doi:10.1080/0 2602938.2015.1102863

Boekaerts, M., Pintrich, P.R. \& Zeidner, M. (2000). Self-Regulation: An Introductory Overview, in M. Boekaerts, P. Pintrich \& M. Zeidner (Eds.). Handbook on Self-Regulation (pp.1-9). San Diego, CA/London: Academic Press.

Boud, D. \& Molloy, E. (Coords.) (2015). El feedback en educación superior y profesional. Comprenderlo y hacerlo bien. Madrid: Narcea.

Brown, S. (2015). A review of contemporary trends in Higher Education. @tic 15, 43-49. doi:10.7203/attic.14.4166

Butler, D. L. \& Winne, P. H. (1995). Feedback and Self-Regulated Learning: A Theoretical Synthesis. Review of Educational Research, 65, 245-281. doi:10.3102/00346543065003245 
Cano, E. (2014). Análisis de las investigaciones sobre feedback: aportes para su mejora en el marco del EEES. Bordón, 66(4), 9-24. doi:10.13042/Bordon.2014.66402

Carless, D. (2016). Diseñar el feedback para promover el diálogo. En N. Cabrera \& R. M. Mayordomo (eds.), El feedback formativo en la universidad. Experiencias con el uso de la tecnología (pp. 13-29). Barcelona: Laboratori de Mitians Interatius. Recuperado de http://www.lmi.ub.es/transmedia21/pdf/9_feedback.pdf

García-Jiménez, E. (2015). La evaluación del aprendizaje: de la retroalimentación a la autorregulación. El papel de las tecnologías. RELIEVE. Revista Electrónica de Investigación y Evaluación Educativa, 21 (2), doi:10.7203/relieve.21.2.7546

Gargallo, B., Suárez-Rodríguez, J.M. \& Pérez-Pérez, C. (2009). El Cuestionario CEVEAPEU. Un instrumento para la evaluación de las estrategias de aprendizaje de los estudiantes universitarios. RELIEVE. Revista Electrónica de Investigación y Evaluación Educativa, 15(2), 1-31. Recuperado de https://www.uv.es/RELIEVE/v15n2/RELIEVEv15n2_5.htm

Gibbs, G. \& Simpson, C. (September, 2003). Measuring the response of students to as- sessment: The Assessment Experience Questionnaire. Paper presented at the 11th International Improving Students Learning Symposium. Hinckley. Recuperado de https://www.semanticscholar.org/paper/Measuring-the-response-of-students-toassessment\%3A-Gibbs-Simpson/57111701f9d713bde7780f6442e6bb4c1cf1b43b

Gibbs, G. \& Simpson, C. (2009) Condiciones para una evaluación continuada favorecedora del aprendizaje. Barcelona: Universitat de Barcelona. Institut de Ciències de l'Educació \& Ediciones Octaedro. Recuperado de http://diposit.ub.edu/dspace/handle/2445/144983

Gudergan, S., Ringle, C., Wende, S. \& Will, A. (2008). Confirmatory tetrad analysis in PLS path modeling. Journal of Business Research, 61, 1238-1249. doi:10.1016/j.jbusres.2008.01.012

Hair, J., Hult, G., Ringle, C. \& Sarstedt, M. (2017). A primer on Partial Least Squares Structural Equation Modeling (PLS-SEM). (2nd. Edition). Los Angeles: SAGE Publications.

Hattie, J. \& Timperley, H. (2007). The power of feedback. Review of Educational Research, 77(1), 81-112. doi:10.3102/003465430298487

Ladyshewsky, R. K. (2015). El papel de los compañeros en los procesos de feedback. En D. Boud \& E. Molly (Coords.). El feedback en educación superior y profesional. Comprenderlo y hacerlo bien (pp. 203-218). Madrid: Narcea.

Lohmöller, J. (1989). Latent variable path modeling with partial least squares. Heidelberg, Germany: Physica.

Margalef, L. (2014). Evaluación formativa de los aprendizajes en el contexto universitario: resistencias y paradojas del profesorado. Educación XX1, 17(2), 35-55. doi:10.5944/ educxx1.17.2.11478

Molloy, E. \& Boud, D. (2015). Cambiar nuestra concepción del feedback, en D. Boud \& E. Molloy, El feedback en educación superior y profesional. Comprenderlo y hacerlo bien (pp. 25-50). Madrid: Narcea.

Nicol, J. \& Macfarlane-Dick, D. (2006). Formative assessment and self-regulated learning: a model and seven principles of good feedback practice. Studies in Higher Education, 31(2), 199-218. doi:10.1080/03075070600572090

Nicol, D. (2015). Resituar el feedback: de reactivo a proactivo, en D. Boud \& E. Molloy, El feedback en educación superior y profesional. Comprenderlo y hacerlo bien (pp. 51-68). Madrid: Narcea. 
Núñez, J.L. \& Reyes, C.I. (2014). La evaluación del aprendizaje de estudiantes: validación española del Assessment Experience Questionnaire (AEQ). Estudios sobre Educación, 26, 63-77. Recuperado de https://www.unav.edu/publicaciones/revistas/index.php/ estudios-sobre-educacion/article/view/1861/1730

Pintrich, R. \& DeGroot, E. (1990). Motivational and self-regulated learning components of classroom academic performance. Journal of Educational Psychology, 82, 33-40. doi:10.1037/0022-0663.82.1.33

Quesada, V., Rodríguez-Gómez, G. \& Ibarra, Mª S. (2013). ActEval: un instrumento para el análisis y reflexión sobre la actividad evaluadora del profesorado universitario. Revista de Educación, 362, 69-104. doi:10.4438/1988-592X-RE-2011-362-153

Salmerón, H., Gutierrez-Braojos, C., Rodriguez, S. \& Salmeron-Vilchez, P. (2011). Metas de logro, estrategias de regulación y rendimiento académico en diferentes estudios universitarios. Revista de Investigación Educativa, 29(2), 467-477. Recuperado https:// revistas.um.es/rie/article/view/116041

Sánchez-Martí, A., Muñoz, J.L. \& Ion, G. (2019). Diseño y Validación de un Cuestionario de Percepción del Aprendizaje a través del Feedback entre Iguales en Educación Superior, Revista Iberoamericana de Diagnóstico y Evaluación Psicológica, 53(4), 113-128. doi:10.21865/RIDEP53.4.09

Sarstedt, M., Ringle, C. M., Henseler, J. \& Hair, J. F. (2014). On the emancipation of PLS-SEM: A commentary on Rigdon (2012). Long Range Planning, 47, 154-160. doi:10.1016/j.lrp.2014.02.007

Snyder, P. \& Lawson, S. (1993). Evaluating results using corrected and uncorrected effect size estimates. Journal of Experimental Education, 61(4), 334-349. doi:10.1080/0 0220973.1993.10806594

Zimmerman, B. (2000). Attaining self-regulation: A social cognitive perspective, in M. Boekaerts, P. Pintrich \& M. Zeidner (Eds.). Handbook on Self-Regulation. (pp. 13-39). San Diego, CA/London: Academic Press.

Fecha de recepción: 17 de abril de 2020.

Fecha de revisión: 7 de mayo de 2020.

Fecha de aceptación: 11 de septiembre de 2020. 


\section{Apéndice I}

\section{Cuestionario sobre feedback en el desarrollo de una tarea}

\section{Estructura de ítems del cuestionario, organizado por dimensiones y sub-dimensiones}

Dimensión 1: Feed-up (conocimiento de las condiciones de realización de la tarea y del sistema de evaluación de la misma)

11. El profesor/La profesora aclaró desde el principio lo que esperaba de sus estudiantes en la realización de la tarea

15. La presentación de la evaluación de la tarea incluyó una explicación de su relación con las competencias que se iban a desarrollar

24. Los criterios de evaluación se expusieron en el mismo momento de presentar la tarea

26. La presentación de la evaluación de la tarea incluyó su relación con los criterios de calificación de la asignatura.

36. Fue fácil comprender los criterios de evaluación con los que era valorada la tarea

44. La presentación de la evaluación de la tarea se relacionó con los contenidos de la asignatura.

Dimensión 2: Feedback

Subdimensión 2.1: Feedback externo

\begin{tabular}{ll}
\hline $\begin{array}{l}\text { Agente que lo pro- } \\
\text { porciona }\end{array}$ & $\begin{array}{l}\text { 16. He recibido feedback de grupos de compañeros y compañeras } \\
\text { 17. He recibido feedback del profesor o profesora } \\
\text { 30. He recibido feedback de un compañero o compañera }\end{array}$ \\
\hline $\begin{array}{l}\text { Solicitado por el } \\
\text { alumnado }^{1}\end{array}$ & $\begin{array}{l}\text { 5. Solicité feedback individualmente y lo recibí } \\
\text { 29. Solicitamos feedback como pequeño grupo y lo recibimos }\end{array}$ \\
\hline $\begin{array}{l}\text { 2. Recibí feedback por escrito a través de e-mail o del aula virtual } \\
\text { 8. Recibí feedback de manera oral de modo presencial }\end{array}$ \\
$\begin{array}{l}\text { 31. Recibí feedback de modo gráfico (sombreando en una rúbri- } \\
\text { ca...) } \\
\text { 38. Recibí feedback por escrito en papel en mano } \\
\text { 43. Recibí feedback de modo oral on line (aula virtual, WhatsApp...) }\end{array}$ \\
$\begin{array}{l}\text { 23. He recibido feedback durante la realización de la tarea } \\
\text { 25. Recibí feedback en el plazo acordado } \\
\text { 42. He recibido feedback después de entregar el primer borrador } \\
\text { 45. He recibido feedback una vez entregada definitivamente la tarea }\end{array}$ \\
$\begin{array}{ll}\text { 32. El feedback fue acompañado de la calificación } \\
\text { Calificación }\end{array}$ \\
$\begin{array}{l}\text { 3. Recibimos feedback como grupo pequeño } \\
\text { 6. Recibimos feedback todo el grupo clase } \\
\text { 46. Recibí feedback individualmente }\end{array}$ \\
\hline
\end{tabular}




\begin{tabular}{ll}
\hline & $\begin{array}{l}\text { 1. He recibido feedback sobre estrategias de trabajo o habilidades } \\
\text { que necesitaba desarrollar }\end{array}$ \\
Tipo de feedback & $\begin{array}{l}\text { 35. He recibido feedback sobre aspectos personales (mensajes de } \\
\text { 4imo, valoraciones sobre capacidades...) } \\
\text { pliar o mejorar }\end{array}$ \\
\hline $\begin{array}{l}\text { Feedback interroga- } \\
\text { tivo }\end{array}$ & $\begin{array}{l}\text { 22. Recibí feedback por medio de preguntas que me hicieron re- } \\
\text { flexionar }\end{array}$ \\
\hline $\begin{array}{l}\text { Feedback entre } \\
\text { iguales }\end{array}$ & $\begin{array}{l}\text { 4. He tenido la oportunidad de ofrecer feedback a mis compañeros } \\
\text { y compañeras de clase y ellos/as conocían mi identidad }\end{array}$ \\
\hline
\end{tabular}

Subdimensión 2.2: Valoración del Feedback

7. Recibí feedback que señalaba de manera específica los aspectos a mejorar

12. Recibí suficiente feedback sobre mi trabajo

14. Me siento satisfecho con el feedback recibido en la tarea

20. Comprendí el feedback que me dio el/la profesor/a sobre mi trabajo

27. Después de recibir el feedback hubo tiempo suficiente para mejorar la tarea antes de la entrega definitiva

33. El feedback que me dieron sobre mi trabajo llegó tarde para ser útil ${ }^{2}$

Subdimensión 2.3: Seguimiento del feedback por parte del docente ${ }^{1}$

28. Al entregar una tarea después de recibir feedback mi profesor/a me pidió que especificara cómo había utilizado los comentarios o feedback recibido

34. Al entregar una tarea mi profesor/a me pidió que especificara aquellos aspectos sobre los que me gustaría recibir feedback

Dimensión 3: Autoevaluación

18. He tenido la oportunidad de autoevaluar la tarea que he realizado

Dimensión 4: Autorregulación

Subdimensión 4.1: Efectos sobre la ARA del feedback recibido

21. El feedback recibido me permitió identificar los aspectos a mejorar de la tarea

39. El feedback recibido me hizo volver a consultar el material que había visto en el curso.

Subdimensión 4.2: Feed-forward

10. El feedback recibido me permitió inferir/aprender claves para aplicar en tareas futuras.

19. A partir del feedback recibido concreté un plan de acción para mejorar la tarea 
Subdimensión 4.3: Efectos sobre la ARA del feedback ofrecido

9. Aportar feedback a mis compañeros me ha permitido afianzar mi comprensión de los contenidos de la asignatura

37. Ofrecer feedback a mis compañeros/as ha hecho que llegue a comprender mejor las demandas de la tarea.

41. Dar feedback a mis compañeros ha favorecido que tenga una actitud crítica ante mis propias producciones/tareas

Dimensión 5: Satisfacción del alumnado

47. Me siento satisfecho con la asignatura ${ }^{1}$

48. Me siento satisfecho con los aprendizajes adquiridos en la asignatura

49. Me siento satisfecho con los aspectos metodológicos de la asignatura ${ }^{1}$

50. Me siento satisfecho con el sistema de evaluación de la asignatura

\footnotetext{
${ }^{1}$ Estos ítems no se han tenido en cuenta en el estudio.

2 Ítem desestimado del modelo PLS-SEM porque, una vez ajustado, el peso sobre la variable reflectiva

"valoración del feedback" era prácticamente igual a 0 .
} 